\title{
Perfil de salud mental, en relación a la depresión, de los estudiantes del 3er semestre de la carrera de medicina en UAEH durante el periodo julio-diciembre 2017
}

\author{
Mental health profile, in relation to depression of the students of the 3rd semester of the medical \\ career in UAEH during the period July-December 2017 \\ María G. Castillo-Oviedo ${ }^{a}$, Mariela Corona-Aguilar ${ }^{a}$, Martín E. Escudero-Hernández ${ }^{a}$, \\ Ximena Moreno-Rodríguez , Ángel E. Oscoy-Hernández, Alejandra Ramírez-González ${ }^{b}$, \\ Brenda E. Barraza Sánchez ${ }^{c}$, Juliana Ramírez-Martínez ${ }^{d}$, Josefina Reynoso Vázquez, ${ }^{e}$,
} Jesús C. Ruvalcaba-Ledezma ${ }^{f^{*}}$.

\begin{abstract}
:
The objective was to describe the true emotional state with respect to depression of the medical students who were studying the third semester to be able to observe the impact that the changes generated when studying this career. Method. This article was carried out through 250 structured surveys of medical students who were in the third semester. Results The majority of the students surveyed do not present a problem, however, attention must be paid since at least $10 \%$ of the respondents could present a mild to moderate depressive episode, which is imperative to recognize individually in order to avoid early attempt of greater negative impact on the health of any student, a possibility that is latent and that nobody really notices it. Conclusion. This study helps to appreciate that being a doctor goes beyond procuring the health of other people and ignoring that of the students themselves, in addition to the fact that depression is often not taken into account, it is thought that it will never be suffered from it, creating an erroneous idea and thanks to this article the opposite is demonstrated.
\end{abstract}

Keywords:

Mental health, depression, medical students, mild to moderate, risk.

\begin{abstract}
Resumen:
El objetivo del presente artículo consistió en describir el estado emocional relacionado con el trastorno de depresión en los alumnos del tercer semestre de medicina para poder determinar los cambios generados al estar estudiando esta carrera. Método. Estudio descriptivo, de tipo inductivo con metodología cuantitativa, mediante la aplicación de 250 encuestas estructuradas a alumnos de medicina que se encontraban cursando el tercer semestre. Resultados. En su mayoría los alumnos encuestados no presentan problemas con su estado emocional, sin embargo, al menos un $10 \%$ de los encuestados se encuentra en riesgo de presentar un episodio depresivo de leve a moderado, lo cual es imperativo reconocer individualmente para evitar de manera temprana mayor impacto negativo en la salud de los alumnos. Conclusión. Este estudio ayuda a apreciar que ser médicos va más allá de procurar la salud de las demás personas e ignorar la de los mismos estudiantes, además de que la depresión muchas veces no se toma en cuenta, se piensa que nunca se padecerá de ella lo que genera una idea errónea y gracias a este artículo se demuestra lo contrario.
\end{abstract}

Palabras Clave:

Salud mental, depresión, estudiantes de medicina, leve a moderado, riesgo.

\footnotetext{
a Área Académica de Medicina, Instituto de Ciencias de la Salud, Universidad Autónoma del Estado de Hidalgo.

b,c Maestría en Salud Pública, Instituto de Ciencias de la Salud, Universidad Autónoma del Estado de Hidalgo. alejandra.go@outlook.com

d Trabajo Social-Área Académica de Medicina, Instituto de Ciencias de la Salud, Universidad Autónoma del Estado de Hidalgo. juliana_ramirez1@outlook.com

Área Académica de Farmacia y maestría en Salud pública, Instituto de Ciencias de la Salud, Universidad Autónoma del Estado de Hidalgo.jreynoso@uaeh.edu.mx

f* Autor de Correspondencia, Instituto de Ciencias de la Salud, Maestría en Salud Pública Universidad Autónoma del Estado de Hidalgo, ORCID:0000-0002-5593-3946, Email: jesus_ruvalcaba@uaeh.edu.mx
} 


\section{Introducción}

Desde que somos pequeños, nuestros padres, amigos y familiares nos hacen una pregunta que marca de alguna manera el destino de tu vida por más sencilla que parezca y esa pregunta es ¿Qué quieres ser de grande? Es fácil contestar: Quiero ser Doctor (a), pero en ese momento no sabemos de qué se trata la carrera, únicamente pensamos que es fácil y es bonito ayudar a las personas sin conocer lo que hay detrás de esta profesión. Nadie te prepara para una carrera profesional, muchos incluso no llegan con la madurez suficiente a esta etapa y los cambios drásticos, relacionados con la carga de materias, los horarios poco flexibles, la cantidad de temas a estudiar, en algunos casos alejarse de la familia para vivir solo en la ciudad, la mala alimentación, el estrés y la preocupación por pasar una materia o salvar el semestre, que se viven se ven reflejados en el estado de salud de los estudiantes; la salud física es notoria: podemos observar que dejamos de hacer ejercicio por las tareas y que nuestra masa muscular ha disminuido pero ¿Cómo nos damos cuenta de que nuestra salud mental está bien?, ¿Cómo darnos cuenta que nuestra salud mental se está deteriorando si no somos capaces de percibir un cambio como en la salud física?. Algo tan sencillo como lo es la depresión puede pasar desapercibida en muchos de los casos y ser diagnosticada hasta un grado más grave.

Según la OMS la depresión es un trastorno mental frecuente, que se caracteriza por la presencia de tristeza, pérdida de interés o placer, sentimientos de culpa o falta de autoestima, trastornos del sueño o del apetito, sensación de cansancio y falta de concentración, que puede llegar a hacerse crónica o recurrente y dificultar sensiblemente el desempeño en el trabajo o la escuela y la capacidad para afrontar la vida diaria. En su forma más grave, puede conducir al suicidio. La depresión no es, como normalmente se le conoce, una situación en la cual sólo te la pasas triste y llorando, abarca muchas más actitudes y genera más problemas de los que se creen. Cuando una persona tiene un trastorno depresivo, este interfiere con la vida diaria y el desempeño normal y causa dolor tanto para quien padece el trastorno como para quienes se preocupan por él o ella. (1)

Por esta serie de razones fue que decidimos conocer más acerca del estado emocional de los compañeros de la carrera de medicina del 3er semestre en el Instituto de Ciencias de la Salud durante el periodo julio-diciembre 2017 realizando una serie de preguntas encaminadas a tener una sospecha de padecer depresión con la finalidad de conocer y dar a conocer la situación real de los alumnos que generalmente pasa desapercibida y puede culminar en la muerte dicha falta de atención. En su mayoría, los estudiantes son adolescentes y otros más adultos jóvenes, siendo una etapa de la vida en la que la depresión puede manifestarse como un comportamiento irritable-desafiante, con diversos trastornos de conducta asociados, entre los que se incluyen el consumo de sustancias psicoactivas, conductas parasuicidas y problemas escolares. (2)

\section{Método}

Es un estudio descriptivo, de tipo inductivo con metodología cuantitativa. Se realizaron 250 encuestas por vía electrónica a alumnos del 3er semestre de la carrera de medicina que van de los 19 a los 24 años. En la encuesta no clasificamos a los participantes por género ni por edad debido a que quisimos generalizar más sobre el estado emocional de todo el grupo de estudiantes, dándoles en común únicamente la misma carrera.

Fueron seleccionados de manera aleatoria, los únicos requisitos que debían de cumplir era ser de tercer semestre y estudiar medicina. Se buscó un lugar con internet para que las encuestas se pudieran hacer sin dificultades.

Se respondieron 10 preguntas relacionadas con su desarrollo individual diario relacionado con la escuela, sus horas de sueño, su apetito, habilidades, capacidad de concentración, emociones, etc.

\section{Análisis de Resultados}

Para este estudio se aplicó un total de 250 encuestas mediante un formulario electrónico. Se incluyeron 10 preguntas cuyo objetivo principal es identificar emociones relacionadas al desarrollo de la depresión. Debido a que es un trastorno que se considera afecta a las personas sin importar sus características sociodemográficas se decidió no estratificar por grupos el análisis de resultados (3).

Se consideró la satisfacción con la carrera elegida como variable independiente del desarrollo de emociones asociadas a la depresión, de acuerdo a los datos obtenidos se observa que el $61.2 \%$ de los participantes se encuentra satisfecho con la elección realizada, $21.6 \%$ en ocasiones ha pensado en cambiar de carrera, $11 \%$ que para nada está satisfecho y $6 \%$ planea cambiar de carrera, aunque se desconoce si los motivos para el cambio están relacionados a las emociones analizadas [Figura 1]. 
Figura1. La satisfacción con la carrera elegida "Medicina".

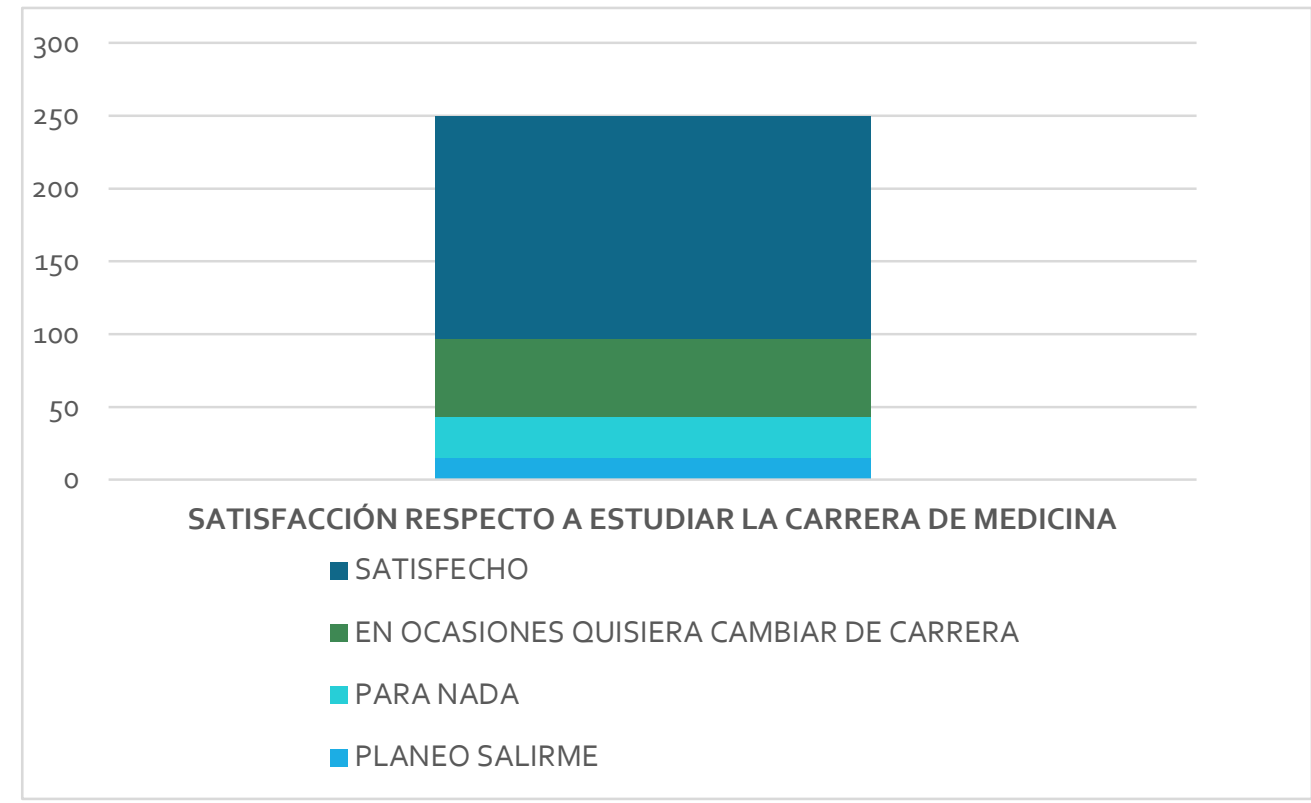

Fuente: Elaboración propia.

Se exploró el estado de ánimo general de los estudiantes, obteniendo como resultado que, 64.4\% presentan un estado de ánimo alegre y optimista, $12.4 \%$ se muestra susceptible ante comentarios, $11.6 \%$ desanimado y triste y $11.6 \%$ enfadado y pesimista. [Figura 2]

Figura 1. Estado de Ánimo en la Escuela.

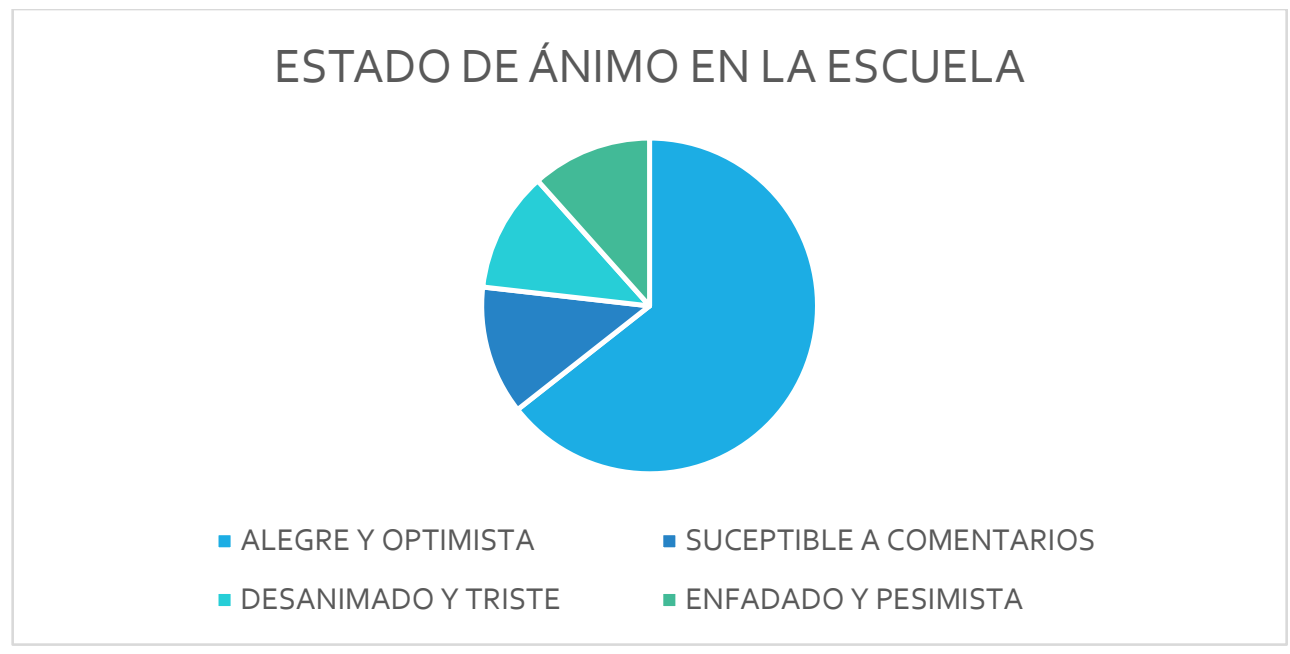

Fuente: Elaboración propia.

En cuanto a los cambios de peso y apetito se reportó que se encuentran presentes en el $79.2 \%$ de nuestros encuestados, este indicador está asociado con cambios de ánimo y está directamente relacionado con la depresión [Tabla 1]. 
Tabla 1. Cambios de peso y apetito de los Alumnos de Medicina.

\begin{tabular}{|c|c|r|r|r|r|}
\hline \multicolumn{2}{|c|}{} & \multicolumn{1}{|c|}{ Frecuencia } & \multicolumn{1}{c|}{ Porcentaje } & Porcentaje válido & \multicolumn{2}{c|}{$\begin{array}{c}\text { Porcentaje } \\
\text { acumulado }\end{array}$} \\
\hline \multirow{3}{*}{ Válidos } & NO & 52 & 20.8 & 20.8 & 20.8 \\
\cline { 2 - 6 } & SI & 198 & 79.2 & 79.2 & 100.0 \\
\cline { 2 - 6 } & Total & 250 & 100.0 & 100.0 & \\
\hline
\end{tabular}

Fuente: Elaboración propia

Se analizaron y cuestionaron factores emocionales que se encuentran asociados al desarrollo de la depresión, identificando que los alumnos que participaron en el estudio mantienen pensamientos de muerte e ideación suicida, $10.2 \%$ siempre y $4 \%$ casi siempre, aunque parecieran porcentajes bajos es importante poner atención en este indicador ya que es un peligro importante para la salud de la población estudiantil. En cuanto al resto de resultados obtenidos, si bien podemos observar que predominan los alumnos que no se sienten identificados con los factores asociados a depresión, existe un porcentaje importante de aquellos que las están experimentando pocas veces o en ocasiones por lo que se debe poner atención inmediata para evitar que conforme avanzan en su desarrollo académico puedan también aumentar estas emociones. [Figura 3]

Figura 3. Factores emocionales asociados a depresión en alumnos de medicina.

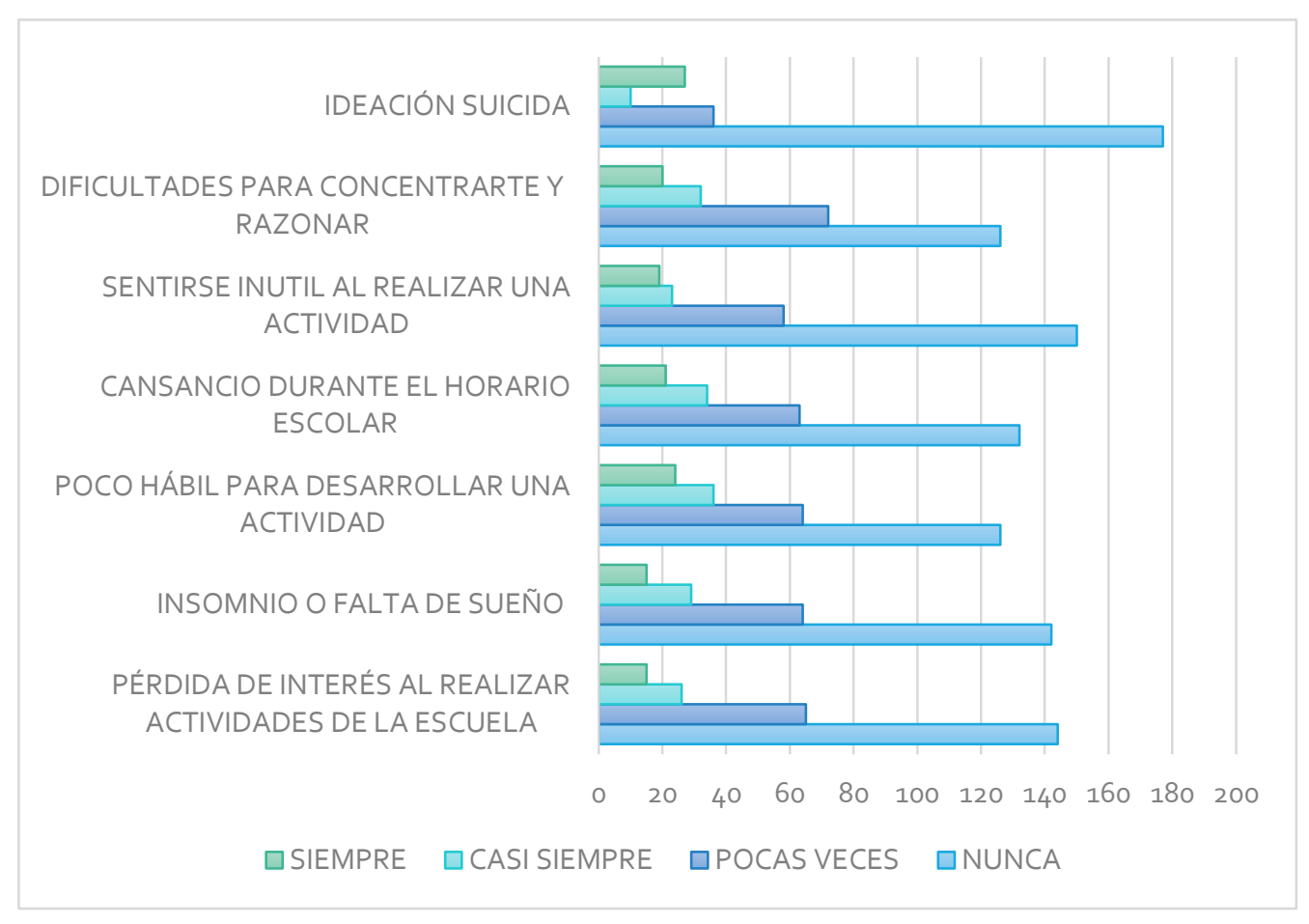

Fuente: Elaboración Propia. 


\section{Discusión}

Al inicio de la investigación teníamos un ligero conocimiento acerca de la situación emocional de los alumnos de 3er semestre basada en simples suposiciones. Después de hacer las encuestas nos dimos cuenta, que muchos de los compañeros presentan datos de depresión leve, que de acuerdo al DSM-V son pocos o ningún síntoma más que los necesarios para cumplir los criterios diagnósticos, la intensidad de los síntomas causa malestar, pero es manejable y los síntomas producen poco deterioro en el funcionamiento social o laboral.

De acuerdo a los criterios diagnósticos de un episodio depresivo según CIE-10 observados en la tabla 1 y según nuestros resultados al menos el $10 \%$ de nuestros encuestados presentan al menos 4 criterios diagnósticos de síndrome somático de un episodio depresivo que pudiera ser leve e incluso moderado y que además de todo pudieran no tener el conocimiento de ello. Es importante mencionar que un diagnóstico certero de la enfermedad es complicado debido a que la depresión es un problema de múltiples facetas caracterizado por un amplio número de síntomas que pueden, o no, estar presentes en cada paciente en particular (4).

Cabe recalcar que el suicidio, un tema controversial y a la vez muy presente en México, como se pudo observar en los resultados, está presente en algunos estudiantes en forma de pensamientos suicidas y muchas veces dichos pensamientos pueden confundir a una persona generando acciones de autolesión. (5)

En el artículo de "Intento de Suicidio en adolescentes mexicanos, se hace una gran referencia a que de los $34.4 \%$ de sus encuestados, con intento de suicido previo, manifiestan depresión, definiéndola ellos mismos como "sin ganas de salir, sin ganas de ver a nadie, no querer hacer nada y no encontrar la salida" (6). Con esto es notorio, que prevenir un caso de depresión puede prevenir de manera considerable un suicidio.

Tabla 1. Criterios diagnósticos de un episodio depresivo

A. El episodio depresivo debe durar al menos dos semanas.
B. El episodio no es atribuible a abuso de sustancias psicoactivas o a trastorno mental orgánico.
C. Síndrome somático: alguno de los síntomas depresivos pueden ser muy destacados y adquirir un sig-
nificado clíico especial. Habitualmente, el síndrome somático se considera presente cuando coexisten al
menos cuatro o más de las siguientes características:
- Pérdida importante del interés o capacidad de disfrutar de actividades que normalmente eran placenteras
- Ausencia de reacciones emocionales ante acontecimientos que habitualmente provocan una respuesta
- Despertarse por la mañana dos o más horas antes de la hora habitual
- Empeoramiento matutino del humor depresivo
- Presencia de enlentecimiento motor o agitación
- Pérdida marcada del apetito
- Pérdida de peso de al menos $5 \%$ en el último mes
- Notable disminución del interés sexual

\section{Conclusión}

Concluimos que este trabajo resultó ser de gran provecho debido a que se pudieron analizar situaciones que van más allá del ámbito académico pero que repercuten de gran manera en él.
Entender la situación real de un grupo de 250 estudiantes de medicina en cuanto a su estado emocional, que siempre pasa desapercibido, es de gran utilidad para ayudar incluso a mejorar su rendimiento académico y aún más importante es detectar trastornos depresivos leves para poder darles un seguimiento y tratamiento oportunos para así evitar que el trastorno 
progrese a uno más severo. Estudiar una carrera relacionada con las ciencias de la salud y con mayor razón, la licenciatura en Médico Cirujano, que como ya sabemos requiere de una gran inversión de tiempo, esfuerzo y compromiso, no significa que se descuidarán los aspectos de salud individuales que a su vez modifiquen el estado emocional.

Es imperante detectar casos de riesgo para poder evitar casos más complicados como, por ejemplo, el suicidio.

\section{Referencias}

[1] Asociación Americana de Psiquiatría. Guía de consulta de los criterios diagnósticos del DSM-5. 5th ed. Estados Unidos; 2014.

[2] Instituto Nacional de Salud Mental. Depresión. EEUU: Institutos Nacionales de Salud, Departamento de salud y servicios humanos de los Estados Unidos; 2009.

[3] Álvarez Ariza M, Atienza Merino G. Guía de práctica clínica sobre el manejo de la depresión en el adulto Madrid, España; 2015.

[4] Alverdi Sudupe , Taboada Ó, Castro DC. Depresión. España:, Servicios de Psiquiatría; 2006.

[5] Gerhard Heinze M, Camacho Segura P. Guía clínica para el manejo de la depresión México: Guías Clínicas para la Atención de trastornos mentales; 2010.

[6] Lahera Forteza G. Las 10 preguntas clave sobre la depresión. Madrid: Universidad de Alcalá de Henares, Psiquiatría y Psicología médica.

[7] López Vázques F, Fonseca Muñoz R, Becoña E. Depresión: Diagnóstico, modelos teóricos y tratamiento a finales del siglo XX España; 2000.

[8] Sánchez Loyo LM, Morfín López T T, Cruz Gaitán JI. Intento de Suicidio en adolescentes mexicanos : perspectiva desde en consenso cultural México: Acta de investigación psicológica; 2014. 